\title{
Prescrição off-label, direito à informação, consentimento informado e processo clinico eletrónico no direito português
}

\author{
Off-labe/ prescription, informed consent and electronical records in Portuguese law \\ Prescripción off-label, consentimiento informado y processo clínico electrónico en la \\ legislación portuguesa
}

\section{Carla Barbosa ${ }^{1}$ \\ Mafalda Francisco Matos ${ }^{2}$}

RESUMO: A prescrição off-label de medicamentos é não só uma prática comum e amplamente aceita, como também incontornável nos sistemas de saúde modernos. Perante a ausência de terapia eficaz, o médico não pode ficar sujeito ao tempo necessário para que todo o processo de aprovação de um determinado uso para um determinado medicamento se desenvolva. No entanto, apesar de se mostrar urgente, ainda não existe uma regulamentação desse tipo de receita médica em Portugal (ou mesmo na Europa). Como uma prerrogativa do médico, a liberdade terapêutica deve garantir o respeito pelo paciente, razão pela qual deve ser plenamente informado. É imperativo que o paciente conheça aquela terapia particular, os seus potenciais riscos e benefícios e as razões que levaram o médico a prescrever off-label. Todas as informações devem, por isso, ser claramente anotadas nos registos clínicos do paciente de modo garantir uma efetiva proteção tanto para o paciente como para o médico. Embora não exista, ainda, consenso quanto à prescrição off-label, em 2014 a Direção Geral de Saúde portuguesa emitiu uma norma que recomenda a obtenção do consentimento informado, sob a forma escrita, para os casos de uso off-label de medicamentos. O problema reside na seguinte questão: como ajustar essa recomendação com a crescente informatização dos registros clínicos e com mecanismos como a Plataforma de Dados de Saúde Português? Na verdade, este é um problema comum. Recentemente, a FDA (Food and Drug Administration, EUA) publicou um projeto que fornece, precisamente, recomendações sobre procedimentos utilizando um consentimento informado eletrónico.

Palavras-Chave: Prescrição de Medicamentos. Prescrição Off-Label. Consentimento Informado. Processo Clínico.

ABSTRACT: Off-label prescription of a medicine is not only common and a widely accepted practice, but also unavoidable in modern health systems. A practitioner tasked with treating patients in this real world, facing a lack of useful therapy, cannot wait for all the procedure to get a licensed medicine use. However, there is still no regulation of this kind of prescription in Portugal (or Europe). As a physician's prerogative, therapy freedom must guarantee the patient's respect, reason why he/she must be fully informed. It is imperative that the patient knows the particular therapy, its potential risks and benefits and the reasons that led the practitioner to prescribe off-label. Besides all information should be

\footnotetext{
1 Investigadora do Centro de Direito Biomédico da Universidade de Coimbra, Coimbra, Portugal. Advogada. E-mail: cbarbosa@fd.uc.pt.

2 Assistente da Faculdade de Direito e Investigadora do Centro de Estudos de Direito Público, Universidade Agostinho Neto, Luanda, Angola. E-mail: mafaldafranciscomatos@gmail.com
} 
comprehensibly noted in the patient's clinical records to ensure protection for both patient and physician.Although there is no consensus about the form of off-label prescription's informed consent, in 2014, an administrative guideline from DGS (Portuguese Health's Direction) recommends a written form. The problem is: how to adjust this demand with the growing informatization of the clinical records and with mechanisms like the Portuguese Health Data Platform? In fact, this is a common health problem. Recently, FDA (U.S. Food and Drug Administration) published a draft guidance that provides recommendations on procedures using an electronic informed consent.

Keywords: Medicine's Prescription. Off-Label. Informed Consent. Electronical Records. Clinical Records.

RESUMEN: La prescripción off-label de medicamentos no sólo es una práctica común y ampliamente aceptado, asi como es esencial en los sistemas de salud modernos. En ausencia de una terapia eficaz, el médico no puede estar sujeto al tiempo requerido para el proceso de aprobación de un uso específico para un medicamento en particular. Sin embargo, aunque es algo urgente, todavía no hay una regulación de este tipo de prescripción en Portugal (incluso en Europa). Como una prerrogativa del médico, la libertad terapéutica debe garantizar el respeto por el paciente, que debe estar plenamente informado. Es imprescindible que el paciente compre su terapia en particular, los posibles riesgos y beneficios y las razones que llevaron al médico que le recete un medicamento off-label. Por lo tanto, toda la información debe estar claramente indicado en el historia clínica del paciente asegurando una protección eficaz tanto para el paciente como para el médico.Aunque todavía no existe un consenso sobre la prescripción off-label, en el año 2014 la Dirección General de Salud de Portugal emitió una norma que recomienda obtener el consentimiento informado en forma escrita, en los casos de uso off-label de medicamentos. El problema radica en la pregunta: cómo ajustar esta recomendación con la creciente informatización de la historia clínica y mecanismos tales como la Plataforma de Datos de Salud portugués? De hecho, este es un problema común. Recientemente, la FDA (Food and Drug Administration, EE.UU.) publicó un proyecto que ofrece precisamente recomendaciones sobre los procedimientos que utilizan un consentimiento informado electrónico.

Palabras-Ilave: Prescripción de Medicamentos. Prescripción Off-Label. Consentimento Informado. Historia Clínica Electrónica.

\section{Introdução}

A prescrição off-label (1) ocorre quando um médico recomenda ou indica um medicamento para um determinado uso que não se encontra previsto na autorização de introdução no mercado (AIM) desse mesmo medicamento. Off-label significa, pois, que a prescrição é feita fora do estabelecido no resumo das características do medicamento (RCM). 
A expressão, que começou por ser usada, sobretudo nos Estados Unidos da América $^{3}$, reflete precisamente essa prática: "Off-label use is a non-approved use. It is the use of a medicine for purpose or indication for which it has not been licensed by the country's medicine authorities." ${ }^{4}$ Quando o FDA (Food and Drug Administration) aprova oficialmente um medicamento, aprova, também, um folheto informativo, específico, para esse medicamento. O label inclui informação relativa às indicações testadas e aprovadas para o uso do medicamento, dosagens, método de administração e população prevista. (2)

Em Portugal, para que um medicamento possa ser comercializado, terá de existir um pedido de autorização de introdução no mercado (AIM) feito ao INFARMED (Autoridade Nacional do Medicamento e Produtos de Saúde I.P.), que zela pela segurança, eficácia e qualidade dos medicamentos de uso humano, acompanhando e garantindo 0 preenchimento de determinados requisitos por parte dos intervenientes no processo de criação do medicamento, desde a fase de investigação até ao seu uso.

Este pedido, apresentado ao INFARMED, faz-se acompanhar pelo resumo das características do medicamento (RCM). É com base no $\mathrm{RCM}^{5}$ que os médicos conhecem a informação que resulta do processo de avaliação do medicamento, maxime as condições e especificidades para as quais o medicamento foi aprovado, fornecendo as indicações necessárias sobre o tratamento com tal medicamento e os seus efeitos ${ }^{6}$.

Sempre que a prescrição médica seja feita de acordo com as características do medicamento, para os usos especificamente indicados, respeitando todos os critérios contidos no RCM ou no folheto informativo, considera-se que a prescrição é feita on-label. Logo que a prescrição seja feita para qualquer outro uso, estaremos perante um caso de off-label (1). Podemos assim dizer, transpondo o conceito de off-label para a nossa realidade, que estamos perante uma 'prescrição fora do RCM'.

O próprio INFARMED alerta para o facto de uma decisão de utilização de um medicamento fora do âmbito em que foi aprovado - por se entender que um dado

\footnotetext{
3 Tomamos como referência a já consagrada preocupação com esta temática nos Estados Unidos da América. Neste caso, a prescrição off-label existe enquanto uso de medicamento não aprovado pelo FDA (Food and Drug Administration).

4 What is "off-label use", Institute for Quality and Efficiency in Health Care in www.informedheathonline.org

5 Deste RCM resulta, também, o folheto informativo (correspondente ao label e conhecido como bula) que acompanha o medicamento e cuja exposição e linguagem deverá ser mais simples e acessível de modo a ser facilmente perceptível pelo público em geral.

6 (www.infarmed.pt)
} 
medicamento se adequa a uma dada indicação terapêutica - face ao caso particular de um doente é da exclusiva responsabilidade do profissional de saúde. De acordo com a Circular Informativa $n$. 184/CD de 12 de dezembro de 2010, o INFARMED informa que "não [lhe] compete (...) pronunciar-se sobre a utilização dos medicamentos para uma indicação terapêutica diferente das que constam nos respectivos RCM".

Resulta claro, pois, que não depende do INFARMED, nestes casos, autorizar essa utilização, uma vez que o medicamento apenas foi testado, avaliado e autorizado nas condições constantes do RCM aprovado7. Estamos, portanto, defronte de uma prática exclusivamente médica, um verdadeiro acto médico ${ }^{8}$.

Para além desta Circular Informativa, que surge no seguimento do famoso caso de uso off-label do medicamento Avastin (bevacizumab) para 0 tratamento da degenerescência macular relacionada com a idade (DMI), só recentemente - e possivelmente, ainda, pelo mesmo motivo - uma norma lançada pela Direcção Geral de Saúde relativamente à exigência de consentimento informado prestado por escrito inclui, numa das suas várias alíneas, "o uso off-label de medicamentos de dispensa hospitalar". Não obstante, já uma notícia de agosto de 2009 dava conta que "os fármacos off-label correspondem a $30 \%$ a $40 \%$ do peso nas vendas em farmácias e de $30 \%$ nos hospitais", como revelava o Centro de Estudos de Medicina Baseada na Evidência (CEMBE) ${ }^{9}$.

Existe, ainda, uma Comunicação aos Estados-Membros da União Europeia, feita pela Comissão das Petições do Parlamento Europeu, que data de 30 de junho de 2004, onde figura no último parágrafo da Resposta da Comissão uma tentativa de definição de prescrição off-label, salientado que esta é uma prática considerada necessária em alguns casos e que se prende, necessariamente, com a liberdade de escolha dos médicos. Não deixa, também, de se referir que será desejável uma minimização deste tipo de prescrição ${ }^{10}$.

\footnotetext{
7http://www.infarmed.pt/portal/page/portal/INFARMED/MEDICAMENTOS USO HUMANO/PRESCRICAO DI SPENSA E UTILIZACAO/MEDICAMENTOS COM AIM

${ }^{8} \mathrm{E}$, dando indícios também deste sentido, mais recentemente, a posição do Acórdão do Tribunal Europeu de Justiça de 11 de Abril de 2013 no Processo C-535/11 (Novartis Pharma GmbH contra Apozyt GmbH) (http://curia.europa.eu/juris/liste.jsf?\&num=C-535/11)

9 (http://farmacia.netfarma.pt)

10 Esta resposta da Comissão de Petições surge da admissibilidade da Petição 813/2003, onde a peticionária sugere a proibição da utilização off-label de medicamentos.
} 
No plano europeu, neste momento, a pressão para que se regule a prescrição offlabel é enorme. Efectivamente, esta preocupação já se fazia sentir em contidas manifestações ${ }^{11}$, sem, no entanto, dar uma resposta clara para o problema em questão. Tal lacuna tem levado a uma proliferação de casos que opõem, entre si, grandes farmacêuticas. Com as recentes alterações legislativas que ocorreram em França ${ }^{12} 13$ e em Itália $^{14}$, a European Federation of Pharmaceutical Industries and Associations (EPFIA) chegou mesmo a emitir uma posição relativamente à promoção de usos off-label. ${ }^{15}$

A Comissão Europeia tem, presentemente, em mãos, um estudo sobre os usos offlabel (Pharm 655 - Study on off-label use ${ }^{16}$ ) do qual ainda não existem resultados ${ }^{17}$.

\begin{abstract}
11 "(...) medication errors do not have an official EU definition yet. The same situation applies to off-label use, and therefore the same obstacles are encountered, when attempting to regulate it. The definition of off-label use is essential and there is a great urge for harmonization, since a common EU definition of off-label use can also provide further guidance to national jurisdictions. There is no available definition of off-label use in Directive 2001/83/EC, however, Directive 2001/82/EC relating to veterinary defines off-label use...", CRUz, Margarida Brito da, "Patient Safety Within Medicinal Products - Medication Errors and Off-Label Use", Lex Medicinae - Revista Portuguesa de Direito da Saúde, N. Especial (IV EAHL Conference), 2014, p. 269

12 França acolheu a "prescription hors AMM" no seu Code de la Santé Publique, através da Loi n.o 2011-2012 du 29 décembre 2011, relative ao renforcement de la sécurité sanitaire du médicament et des produits de
\end{abstract} santé.

${ }^{13}$ EPFIA: French legislation undermines EU regulatory framework by endorsing off-label use of medicines for economic reasosn (http://www.efpia.eu/mediaroom/189/43/EFPIA-French-legislation-undermines-EUregulatory-framework-by-endorsing-off-label-use-of-medicines-for-economic-reasons)

14 Vide Legge 8 aprile 1998, n. 94, Gazetta Ufficiale n. 86 del 14 aprile 1998. Cfr., também, GuIDI, Benedetta; Nocco, Luca, The debate concerning the off-label prescriptions of drugs: a comparison between Italian and U.S. law, Op. J., Vol. 1/2011, Paper n.1, p.6 (http://lider-lab.sssup.it/opinio): "Yet, the above-mentioned Law no. $244 / 2007$ seem to have partially modified the requirements for off-label prescription, which where previously introduced by Law n. ${ }^{\circ}$ 94/1998". Se, até então, a situação era pacífica, as recentes notícias dão conta da violação das disposições europeias: "Promotion of off-label use of medicines by European healthcare bodies in indications where authoried medicines are available" (http://www.efpia.eu/documents/106/48/Promotion-of-off-label-use-of-medicines-by-European-healthcarebodies-in-indications-where-authorised-medicines-are-available); "Statement on bio-pharmaceutical industry complaint to the European Commission against a new Italian law promoting off-label use of medicines for economic reasons" (http://www.efpia.eu/mediaroom/234/43/Statement-on-bio-pharmaceutical-industrycomplaint-to-the-European-Commission-against-a-new-Italian-law-pPromoting-off-label-use-of-medicines-foreconomic-reasons)

15 EUROPEAN FEDERATION OF PHARMACEUTICAL INDUSTRIES AND AsSOCIATIONS, "Promotions of off-label use of medicines by European healthcare bodies in indications where authorised medicines are available", Position Paper, $\quad$ EPFIA $\quad-\quad$ May 2014 (http://www.efpia.eu/uploads/EFPIA Position Paper Off Label Use May 2014.pdf)

16 "a. Scope of the study: The study intends to cover two aspects: - a scientific one regarding the public health aspects related to the off-label use of medicinal products and in particular patient safety; - a legal one regarding the regulatory framework for the off-label use of medicines. The study would gather information in order to identify if there is a need for coordination at EU level and, if so, possibly, to what extend.", European Commission, Pharmaceutical Committee, 26 March 2014, PHARM 655, Study on off-label use, (http://ec.europa.eu/health/files/committee/72meeting/pharm655.pdf)

${ }_{17}$ A este propósito - salpicado de ironia - o interessante artigo da PharmExec.com, "Europe: Putting Off Offlabel Decisions", de 16 de Março de 2015, conclui do seguinte modo: "The current Comission position is comfortably hands-off on the question. EU legislation on medicines does not regulate off-label use. "It is the 
Enquanto não existe uma definição, em termos que nos parecem concretos, identificamos a prescrição off-label como o uso de medicamentos, por indicação médica, que não se encontra aprovado ou licenciado pela autoridade responsável no país em causa, não podendo, por isso, ser garantida a sua segurança, fiabilidade ou eficácia, dado que não passou pelos necessários testes e requisitos básicos para que a sua comercialização com essa finalidade seja perfeitamente admitida. Não esqueçamos, obviamente, que é o facto de se encontrar aprovado pela autoridade competente que confere segurança ao uso de medicamentos.

O INFARMED e a Agência Europeia do Medicamento (European Medicines Agency EMA $)^{18}$ dispõem de comissões especializadas, nomeadamente da Comissão de Avaliação de Medicamentos, às quais compete emitir pareceres relativos a matérias que se prendam com a qualidade, segurança e eficácia dos medicamentos no âmbito das AIM. Todas as indicações terapêuticas constantes nos RCM foram objecto de apreciação e aprovação por parte destas entidades e reflectem os dados apresentados aquando do processo de avaliação do medicamento.

\section{Os diferentes tipos de prescrição off-label}

A prescrição off-label reveste-se de várias formas de efectivação e não se lhe poderá, para já, atribuir um significado unívoco. Pode considerar-se off-label quando a prescrição é feita fora das indicações terapêuticas, quando não é respeitada a posologia ou dosagem recomendada, se se altera a duração do tratamento ou, ainda, se é destinada a um doente fora do grupo previsto como destinatário ${ }^{19}$.

Além desta diversidade, podemos, ainda, estar perante medicamentos para os quais já há evidências médicas para indicações não registadas (não existindo, contudo,

\footnotetext{
marketing authorization that defines the approved indications", say Commission officials. "Any departure from those terms will remain, in most member states, the responsibility of the prescribing physician." In other words, don't come to us. If you've got a problem, talk to the member states. And if member states have a problem, then they will have to talk to doctors. But leave us alone. We've started a study. What else do you want?" (http://www.pharmexec.com/europe-putting-label-decisions)

18 A EMA tem emitido alguns pareceres e tem tomado em consideração situações de uso off-label de medicamentos. Cfr. Questions and answers on the potential off-label use of celecoxib in patients with familial adenomatous poluposis (EMA/CHMP/376406/2011); Guideline on good pharmacovigilance practices Module VI, Manegement and reporting of adverse reactions to medical products) (EMA/873138/2011)

${ }^{19}$ No mesmo sentido de BEGUE, D., in La precription de médicaments hors AMM, Médecine et Droit, $n^{\circ}$ 60, 2003, Elsevier (www.sciencedirect.com).
} 
verdadeiramente, um interesse da indústria em proceder a esse registo, mas a sua recomendação é amplamente aceite e generalizada) ou poderá tratar-se de fármaco para o qual a evidência ainda não é sobejamente conhecida e comprovada e cujo uso depende inteiramente daquela prescrição médica ${ }^{20}$. No entanto, não devemos retirar precipitadas conclusões, uma vez que um medicamento que seja habitualmente recomendado através da sua prescrição off-label, poderá não ser vantajoso naquele caso concreto.

Podemos, ainda, considerar que existe uma divisão da prescrição off-label na medida em que podemos ter uma recomendação de um medicamento para uma doença com sintomas completamente diferentes daquela para a qual o uso se encontra aprovado, ou, por outro lado, a prescrição de um medicamento para tratar uma doença ou condição para a qual tenha sido aprovado, mas alterando algumas especificações. Claro que, à partida, o primeiro tipo de caso é mais problemático e gera maiores dúvidas do que o segundo, que é, naturalmente, mais aceitável. No entanto, em termos práticos, não existe um estudo comprovado que possa assegurar que um dos casos seja mais seguro que outro ${ }^{21}$.

Importa, porém, estabelecer uma clara distinção entre a prescrição off-label e uma outra realidade com a qual esta pode, por vezes, ser confundida. (3) ${ }^{22}$. É que, de facto, podemos correr o risco de colocar a prescrição off-label numa área experimental, onde a sua definição não lhe permite estar.

Os ensaios clínicos e o uso experimental ${ }^{23}$, ainda que constituindo actividades próprias do processo de uso de medicamentos, não têm o mesmo objectivo desta prescrição. De resto, estas são fases, normalmente, anteriores ao processo de entrada do medicamento no mercado.

É verdade que este tipo de prescrição é demasiado subjectiva, mas não podemos atribuir-lhe uma classificação de experimentação. Não, pelo menos no sentido de experimentação pura, que acontece nos outros casos referidos e que os exclui de um acto médico por não se pretender curar ou satisfazer interesses individuais do doente, mas

\footnotetext{
20 Conforme compreendemos do estudo do Relatório da Oficina sobre Ações Judiciais para o Acesso a Medicamentos: As Demandas por Medicamentos Importados e de Pesquisas Clínicas, de 23 de outubro de 2003, Escola Nacional de Saúde Pública Sérgio Arouca, Fiocruz, Rio de Janeiro

21 "Off-label" Drug Use" (http://www.consumerrports.org/health/resources/pdf/best-buy-drugs/money-savingguides/english/off-label-FINAL.pdf)

22 "It is not always clear whether a physician's prescription of a drug for off-label use is experimental research."

${ }^{23}$ Sobre os quais não nos deteremos, sendo a referência aos mesmos feita, apenas, para que possamos distinguir a actividade experimental da efectiva, prescrição off-label.
} 
antes interesses gerais e comunitários que relegam o doente para uma posição passiva (3). Na prescrição off-label, assiste-se a uma efectiva intervenção terapêutica, a uma intenção de debelar a doença que aquele doente, em concreto, apresenta. Neste sentido, afirma-se que a diferença substancial entre estes dois campos reside no interesse preponderante que o médico assume aquando do aconselhamento feito para o uso do medicamento. Se em causa estiver, como já referimos, um interesse supra-individual de obter conhecimento geral, estamos perante uma área experimental. Ao invés, se a prescrição é feita para obter um benefício directo para aquele paciente concreto, procurando uma efectiva melhoria da sua saúde e bem-estar, motivada tão só pelo interesse do doente, aí já entramos no campo terapêutico e estaremos em sede de um uso off-label do medicamento ${ }^{24}$.

A justificação ética para a prescrição off-label assenta, precisamente, na necessidade de permitir o uso da melhor terapêutica possível para aquele doente concreto. $O$ que contrasta, claramente, com a justificação ética sobre a qual se apoiam os ensaios clínicos e que passa, naturalmente, pelo desenvolvimento de novas terapias para futuros pacientes (2).

A prescrição off-label, podendo ocorrer em qualquer área da medicina, é particularmente comum na Pediatria, na Geriatria, na Oncologia e, por maioria de razão, em doenças raras e consideradas 'órfãs' de terapêutica, onde esta prescrição tem todo o sentido e pertinência.

Um dos casos que, dentro das realidades que estudámos, maior preocupação suscita é, sem dúvida, o da Pediatria. A maior parte das prescrições feitas para crianças acontece fora do que se encontra estudado, regulamentado, com provas de segurança e eficácia dadas. A verdade é que mais de $50 \%$ dos medicamentos usados em crianças não foram objecto dos estudos e testes necessários para que se possa permitir a sua entrada no mercado. (6)

Esta situação acontece, sobretudo, fruto da dificuldade de obtenção de consentimento para que se possam fazer tais intervenções sobre os menores, incapazes de prestarem aquele consentimento por si, e, também, por uma questão financeira, dado

\footnotetext{
${ }^{24}$ Adoptando a ideia de Mehlman: "(...) the key is the physician's intent: if the intent is primarily to benefit the patient, the intervention is therapy. If the intent is solely to test an hypothesis and obtain generalizable knowledge, the interventions is an experiment".
} 
que a panóplia de testes necessária para que o medicamento seja aprovado para um uso pediátrico irá acrescer ao já muito dispendioso processo para aprovação para o uso em adultos. Deste modo, na maior parte dos casos, não resta ao médico senão a prescrição através da adaptação da dosagem que é recomendada para adultos, sob pena de não ter qualquer outra terapêutica para aplicar. Outras vezes, a solução passa, já sobre o âmbito do uso não licenciado, pelo uso de medicamentos manipulados, de modo a combater a falta de possibilidades comercializadas para uso especificamente pediátrico (7).

Não obstante, importa não esquecer que as crianças não são adultas em miniatura e que não thes pode ser aplicada uma "regra de três simples" (6). Parece-nos, aqui, que, na maioria dos casos, existe um risco que não deveria ser corrido, já que estamos a falar de uma população especial, que carece da maior atenção e para a qual a ausência de fármacos acontece por motivações principalmente econômicas. Motivações economicistas que obrigam a ter na mira crítica as indústrias farmacêuticas e os próprios Estados. $\mathrm{Na}$ realidade, esta ausência de medicação específica, importa que se deixe claro, não acontece - como vemos suceder nos demais casos - por qualquer desfasamento entre a evolução dos conhecimentos médicos e científicos e a aprovação de novas, mais sofisticadas e eficientes terapêuticas

\section{A prescrição off-label e o princípio da liberdade terapêutica}

Um dos princípios fundamentais da prática médica é, sem dúvida, a liberdade terapêutica. Acreditamos, por isso, que a prescrição off-label tem o seu fundamento, precisamente, nessa faculdade do médico.

A liberdade terapêutica encontra-se, desde logo, plasmada no próprio Código Deontológico dos Médicos, quando se confere a estes profissionais independência, autonomia, isenção e liberdade nas suas escolhas terapêuticas, nas suas prescrições, de acordo com a ciência e a sua consciência (7).

A esta liberdade terapêutica liga-se, neste sentido, a liberdade de prescrição. $O$ médico é livre de prescrever aquele que considera ser o melhor tratamento para a saúde do seu paciente, para o melhor benefício do mesmo e é, pois, nesta medida que se justifica que exista uma prescrição como a que é feita a título off-label. Esta prescrição deve ser 
feita de forma livre e serena, não se encontrando motivada por influências e vicissitudes estranhas à saúde daquele paciente concreto.

Ademais, a prescrição, assim entendida, surge de uma mudança paradigmática no conceito de acto médico e na relação médico - doente.

A actividade médica deve, pois, guiar-se harmoniosamente pelo melhor bem-estar e benefício do doente. A primazia concedida ao interesse do doente leva a que hoje se abandone o tradicional estereótipo de relacionamento entre o médico e o paciente, caracterizado por certo paternalismo, que excluía a livre vontade deste último (9). Só com este entendimento do acto médico se pode justificar a existência de uma prescrição offlabel. Há um abandono do paternalismo para que, no exclusivo interesse do doente, se obtenham os melhores resultados da prática médica.

Poder prescrever o melhor tratamento possível, adaptando-o a um paciente que será, necessariamente, diferente de todos os outros, constitui para o médico uma certeza de que estará a optar pelo melhor de entre o cientificamente e tecnicamente possível (8). Esta liberdade não pode, porém, ser um poder atribuído ao médico, sem quaisquer limitações. Deverá existir uma escolha reflectida na prescrição, adoptando-se uma correcta proporção entre o benefício do doente e os dados científicos existentes. A prescrição terá de ser feita com enorme precaução e de acordo com a melhor consideração do risco/ benefício. A prudência deve constituir, sempre, um elemento moderador da liberdade de prescrever.

Nos casos em que é necessário efetuar um uso off-label do medicamento, haverá, necessariamente, à partida, uma situação excepcional. Prescrever fora do que se encontra admitido, testado, licenciado, constitui um risco que só pode ser justificado pela excepcionalidade do caso.

Nessa conformidade, o interesse do paciente em obter o melhor tratamento possível para combater a doença terá de ser o princípio, o fundamento e o limite da prescrição. $O$ interesse do paciente deverá constituir uma dupla limitação à autonomia de prescrição do médico (8). Por um lado, o médico não poderá prescrever senão de forma conscienciosa, lançando mão de todos os conhecimentos científicos de que dispõe, com respeito pelas leges artis. Com a certeza, porém - e daí que se suscite a questão do off-label -, que esses conhecimentos e dados científicos se encontram em constante mudança e, portanto, de que se pede ao médico que mantenha os seus conhecimentos actuais, correspondendo àquilo que se espera de um profissional, pelo menos, normalmente informado e diligente. 
A inovação terapêutica só é oportuna, neste sentido, quando os conhecimentos testados, já comprovados e aprovados se mostrem insuficientes. A consciência do médico deve funcionar como uma balança onde num prato é colocado o risco e noutro o benefício da terapêutica que irá usar. Terá de se verificar uma actuação proporcional e o interesse do doente deverá levar o médico a abster-se de lhe proporcionar um risco injustificado e um consequente dano injustificado ${ }^{25}$ (5). É o benefício particular e individual do paciente que prepondera nesta avaliação, o que vale por dizer, no fundo, que terá de existir uma fundamentação bastante válida para se entrar numa melindrosa terapêutica off-label, para que o risco seja permitido.

\section{O Consentimento informado}

\section{A informação de saúde - direito à informação e direito de acesso}

A Constituição da República Portuguesa consagra, desde logo, no seu artigo 25.․, o direito universal à inviolabilidade da integridade moral e física; este conceito resulta, por outro lado, otimizado na Lei de Bases da Saúde ${ }^{26}$, na Base XIV, com o direito a ser informado sobre a situação, as alternativas possíveis de tratamento e a evolução provável do seu estado, permitindo, assim, a decisão de receber ou recusar a prestação de cuidados que é proposta.

Também a Convenção de Oviedo, no seu artigo 5.․, determina, com a sua regra geral que: a) $\mathrm{O}$ âmbito do consentimento livre e esclarecido diz respeito a "qualquer intervenção no domínio da saúde"; b) o sujeito do consentimento é a pessoa a quem são prestados os cuidados e não outros como os seus familiares, salvo as exceções, previstas no art.․ 6.027 ; c) o âmbito do conteúdo da informação a transmitir (objetivo; natureza e consequências do ato/ intervenção proposto).

O consentimento informado, livre e esclarecido da pessoa é, assim, e desde logo, uma manifestação de respeito pelo próprio ser humano, quer este se encontre doente ou não. Reflete, em particular, o direito moral da pessoa à integridade corporal e o direito de autonomia na participação ativa da tomada de decisões conducentes à manutenção da sua

25 "From a legal and ethical standpoint, off-label use represents a delicate balance between the regulatory objective of protecting patients from unsafe or ineffective drugs and medical devices on the one hand, and, on the other hand, the prerogative of physicians to use their professional judgment in treating patients."

26 Lei de Bases da Saúde (Lei n. 0 48/90, de 24 de agosto).

${ }^{27}$ Relativo às pessoas que careçam de capacidade para prestar o seu consentimento. 
saúde e à própria adesão à terapêutica, o que pressupõe claro está, a adequada informação e uma decisão livre e esclarecida (10).

A Norma 15/2013 da Direção Geral de Saúde esclarece que

o consentimento informado no âmbito da saúde emana da atenção dada ao princípio ético do respeito pela autonomia, em que se reconhece a capacidade da pessoa em assumir livremente as suas próprias decisões sobre a sua saúde e os cuidados que the são propostos. Implica a integração da pessoa no processo de decisão quanto aos atos/intervenções de saúde e para a participação em estudos de investigação que the são propostos, numa partilha de conhecimentos e aptidões que a tornem competente para essa decisão de aceitação ou recusa dos mesmos.

Acrescenta, ainda, que "mais do que uma lição formal tendente à obtenção de uma assinatura, na forma escrita, deve constituir um momento de comunicação efetiva, numa lógica de aumento da capacitação da pessoa, fornecendo-lhe as ferramentas necessárias à decisão que vier a assumir, seja numa intervenção de saúde, seja na participação numa investigação, assumindo que os princípios basilares da beneficência, em que a proposta do ato surge a bem do doente, e da não maleficência, que implica a ponderação do risco, estão salvaguardados". E conclui que

a obtenção do consentimento informado deve passar por um processo pelo qual se pretende promover o máximo de confiança possível da pessoa na relação a construir, a fim de se conseguir um maior sucesso terapêutico. $O$ resultado final resultará da interação de entre ambos, numa ponderação entre o possível e o aceitável para ambas as partes. Esta lógica negocial é por definição contínua, não se esgotando no momento da aceitação, mas prolongando-se num tempo útil definido em cada caso pela situação de saúde, em apreço ${ }^{28}$.

Claro que este direito à informação pressupõe, por parte do médico, uma explicitação clara e suficiente, bem como

a disponibilidade para esclarecer as questões que the sejam solicitadas pelo paciente e, por banda deste, a compreensão e colaboração manifestada na prestação àquele de informação completa e verdadeira. Verdade e suficiência são os dois requisitos fundamentais do necessário diálogo cujo objectivo é a compreensão,

28 “Norma da Direção-Geral de Saúde N.o 015/2013, de 3/10/2013, actualizada a 14/10/2014 (www.dgs.pt) 
e que deve ser estabelecido entre o agente médico e a pessoa que aceita, pretende ou necessita a intervenção daquele na sua esfera físico-psíquica (11).

Importa, neste domínio, estabelecer a distinção entre aquilo que é considerado informação/esclarecimento terapêutico, considerado como a "comunicação de todas as informações necessárias para que o paciente cumpra devidamente uma prescrição ou se prepare para uma intervenção diagnóstica ou curativa", da chamada informação/esclarecimento para a autodeterminação em que estamos "perante a informação que o médico deve dar previamente a qualquer intervenção médica, em ordem a permitir a livre decisão do paciente e para dar cumprimento ao princípio da autonomia da pessoa humana" (10).

A propósito do direito à informação, Guilherme de Oliveira entende que devemos adotar o critério do paciente concreto. Refere o autor que "em bom rigor, a necessidade de informação deveria chegar ao ponto de considerar aspetos irrelevantes para o comum dos doentes, mas que são importantes para o paciente concreto... esta exigência seria apenas um corolário do propósito básico de obter um consentimento esclarecido do doente concreto que está perante o médico" (12). Já André Dias Pereira, no mesmo sentido, assume a adoção do padrão do paciente concreto, de um tal modo "que há o dever jurídico do médico conduzir o diálogo por forma a apurar os caracteres básicos da personalidade e da capacidade cognitiva do paciente", acrescentando, inclusivamente, que "uma informação em abstracto perfeita pode ser inútil se o paciente não perceber o idioma ou for analfabeto, se não tomar em consideração a religião ou as opções filosóficas profundas do paciente, ou se as consequências laterais ou os riscos, sendo desprezíveis para um paciente abstracto, forem insuportáveis para o concreto paciente, devido à sua profissão" (10)

Do que vem de ser dito resulta claro que o paciente é o titular da sua informação de saúde e leva, necessariamente a que se lhe reconheça o direito ao respeito pela sua vida privada e o direito a aceder à sua informação. Em 1997, quando a Convenção sobre os Direitos do Homem e da Biomedicina ${ }^{29}$ - também designada por Convenção de Oviedo -

\footnotetext{
${ }^{29}$ Aberta à assinatura dos Estados Membros em Oviedo, em 4 de abril de 1997, aprovada para ratificação por Resolução da Assembleia da República, em 19 de outubro e ratificada pelo Decreto do Presidente da República $n .0$ 1/2001, de 3 de janeiro. O Conselho Nacional de Ética para as Ciências da Vida refere que a Convenção sobre os Direitos do Homem e a Biomedicina é "um marco importantíssimo na história universal
} 
foi aberta à assinatura dos Estados membros do Conselho da Europa, um dos assuntos mais pacíficos, sobre o qual houve consenso, foi, precisamente, a tutela da vida privada e 0 acesso à informação médica ${ }^{30}$.

Dispõe a Convenção, no seu artigo $10{ }^{\circ}$, que "qualquer pessoa tem direito ao respeito da sua vida privada no que toca a informações relacionadas com a sua saúde", reforçando dessa forma a ideia de que os dados de saúde fazem parte da vida privada, não estando no âmbito da esfera da vida pública e, portanto, susceptíveis de conhecimento pelo público em geral ${ }^{31}$.

dos Direitos Humanos pois que pela primeira vez se consegue reunir, num texto consensual, princípios gerais e disposições específicas relativas à protecção do ser humano face à possível utilização indevida da Biologia e da Medicina", alertando que "pela primeira vez num texto de uma Convenção, tenta-se estabelecer os equilíbrios justos mas difíceis entre os direitos e os interesses do indivíduo, da sociedade, da ciência e da espécie humana". Ainda para o Conselho Nacional de Ética para as Ciências da Vida, "a consciência de que ao ser humano é devido respeito tanto como indivíduo e como pertencente à comunidade humana ou como membro da sociedade e que entre eles se estabelece uma teia de direitos e deveres com hierarquias distintas ("o interesse e o bem-estar do ser humano devem prevalecer sobre o interesse único da sociedade ou da ciência" - artigo $2^{0}$ desta Convenção) merece o nosso mais profundo aplauso" (Parecer 30/CNECV/2000, disponível em www.cnecv.gov.pt).

30 Importa referir que, não obstante os conceitos de privacidade e confidencialidade estarem interligados, eles possuem significados diferentes. Enquanto a privacidade diz respeito ao direito a ficar sozinho, ao direito a impedir intrusões de terceiros, a confidencialidade prende-se com o direito de cada um a proteger as suas informações de revelações indesejadas. Enrique Costas define a confidencialidade como "el recinto acorazado que envuelve y protege el secreto personal participado, la camara segura donde ese secreto puede reposar con-fidencia, confiado", dizendo ainda que "los limites del secreto van, por tanto, más allá del secreto, comprenden su periferia: aquellos detalles, referencias, métodos e instrumentos que, aun siendo en sí mismos inocuos, pudieran dejar traslucir el secreto o deslizar su revelación", CoSTAS, Enrique, "Confidencialidad y ficheros de datos", in Jano, 1991; XLI, n.ำ 20.

31 A Convenção de Oviedo aborda especificamente a proteção da vida privada quanto aos dados de saúde. Porém, a proteção da vida privada em geral resulta de outros diplomas internacionais como a Declaração Universal dos Direitos do Homem (artigo 12. - "Ninguém sofrerá intromissões arbitrárias na sua vida privada, na sua família, no seu domicílio ou na sua correspondência, nem ataques à sua honra e reputação. Contra tais intromissões ou ataques toda a pessoa tem direito a protecção da lei."), a Convenção Europeia dos Direitos do Homem (artigo 8.․ - "Qualquer pessoa tem direito ao respeito da sua vida privada e familiar, do seu domicílio e da sua correspondência), o Pacto Internacional de Direitos Civis e Políticos (artigo 17.․ "Ninguém será objecto de ingerências arbitrárias ou ilegais na sua vida privada, na sua família, no seu domicílio ou na sua correspondência, nem de ataques ilegais à sua honra e reputação.") ou a Declaração Universal sobre Bioética e Direito Humanos da UNESCO (artigo 9.- A vida privada das pessoas em causa e a confidencialidade das informações que lhes dizem pessoalmente respeito devem ser respeitadas. Tanto quanto possível, tais informações não devem ser utilizadas ou difundidas para outros fins que não aqueles para que foram coligidos ou consentidos, e devem estar em conformidade com o direito internacional, e nomeadamente com o direito internacional relativo aos direitos humanos). A propósito do artigo 9. da Declaração Universal sobre Bioética e Direitos Humanos da UNESCO, no documento explicativo da elaboração da Declaração, de Abril de 2005, o conteúdo do norma é assim desenvolvido: "A right to privacy guarantees a control over personal information in many ways. It restricts Access to personal and medical information and it provides a claim of non-interference in various private spheres of the individual. Privacy extends beyond data protection, as certain private spheres of the individual that are not manifested in data processing can also be protected by the right to privacy", dizendo-se ainda que "Confidentiality refers to a special and often fiduciary relationship, such as that between researcher and research subject, or doctor and patient, and provides that the shared information shall remain secret, confidential and shall not be disclosed to 
No caso do direito interno português, os dados de saúde inserem-se numa esfera muito restrita da vida privada que, a Constituição da República Portuguesa, através do seu artigo $26 .{ }^{\circ 2}$, tutela como um direito fundamental ${ }^{33}$ : o direito à reserva da intimidade da vida privada e familiar (13) ${ }^{34}$.

É comum dizer-se que o conteúdo deste direito respeita a dados muito sensíveis (14) 35 ou pessoalíssimos, na medida em que neles se encontra envolvido o último reduto da vida privada ${ }^{36}$.

Paulo Mota Pinto escreve, a este propósito, que "os elementos respeitantes à saúde, tais como, por exemplo, a história clínica da pessoa, integram também, sem dúvida, a vida privada protegida", recordando que "no Acórdão n. ${ }^{\circ} 355 / 97$, embora para chegar a uma conclusão de inconstitucionalidade orgânica (já que a regulamentação da matéria em questão, relativa aos "direitos, liberdades e garantias", estava reservada à Assembleia da República), o Tribunal Constitucional afirmou que "o tratamento autonomizado de dados relativos a doenças oncológicas integra-se na esfera de privacidade dos doentes, interferindo, nessa medida, na definição do conteúdo da vida privada, matéria respeitante a direitos, liberdades e garantias". A conclusão, segundo o autor, é a de que

third persons, unless a strictly defined, compelling interest justifies disclosure under domestic law." (texto disponível em htttp://unesdoc.unesco.org).

$32 \mathrm{O}$ direito à reserva da intimidade da vida privada e familiar, assim como os outros direitos fundamentais previstos no artigo $26 . \circ$, são direitos concretizadores daqueloutro direito mais abrangente que é o respeito pela dignidade humana.

${ }^{33}$ Direitos fundamentais que apresentam, como se sabe, como principais características o facto de se aplicarem de forma imediata, vincularem todos os sujeitos de direito (públicos e privados), admitirem apenas restrições impostas por lei e na medida do necessário para salvaguardar outros direitos e interesses constitucionalmente tutelados.

${ }^{34} \mathrm{O}$ direito à intimidade da vida privada e familiar se pode analisar em dois outros direitos menores: o direito de impedir o acesso de estranhos a informação sobre a vida privada e familiar e o direito a que ninguém divulgue as informações que tenha sobre a vida privada e familiar de outrem.

35 Romeo-Casabona define os dados sensiveis como "data in connection with which the data subject is more vulnerable when the data is known or used by a third party because of its potential for causing discrimination and other misuse, especially when accessed, used or illicitly disclosed"

${ }^{36}$ A propósito da reserva da vida privada e da proteção de segredo médico poderíamos analisar aqui muitas outras normas nacionais e supranacionais. Não o fazemos de forma consciente pois saberíamos que ao analisar essas normas teríamos de desenvolver o estudo de temas como a privacidade, os dados pessoais e o segredo médico. Não é possível analisar a problemática do acesso ao processo clínico sem tocar nestes temas, no entanto, apenas pretendemos abordá-los de forma reflexa. Indicamos, contudo, algumas das normas que versam sobre estas problemáticas: Código Civil (artigo 80.), Código Penal (artigo 195.), Lei de Bases da Saúde (Base XIII e XIV), Regime Geral dos Arquivos e Património Arquivístico (artigo 17.‥ n.ำ 2), Estatuto da Ordem dos Médicos (artigo 13.․, alínea c)) e o Código Deontológico da Ordem dos Médicos (artigos 85. a 93.) entre outras. 
os dados de saúde integram a categoria de dados relativos à vida privada, tais como as informações referentes à origem étnica, à vida familiar, à vida sexual, condenações em processo criminal, situação patrimonial e financeira (...), fazem parte da vida privada de cada um. (15)

Contudo, convém ter presente, desde já, que o artigo 10. da Convenção de Oviedo no seu $n$. - 2 afirma que "qualquer pessoa tem o direito de conhecer toda a informação recolhida sobre a sua saúde", ressalvando que "a vontade expressa por uma pessoa de não ser informada deve ser respeitada", e, no n. ${ }^{\circ} 3$, que "a título excepcional, a lei pode prever, no interesse do paciente, restrições ao exercício dos direitos mencionados no $\mathrm{n} .{ }^{\circ}$ 2".

A Convenção de Oviedo, com esta opção, estabelece como regra o direito de informação e acesso aos dados de saúde por parte do titular, admitindo como exceção aquelas situações em que não seja benéfico aos interesses do paciente o exercício desses direitos.

Estas opções positivadas apresentam um claro corte com "a velha tradição de paternalismo clínico" (16). O facto de se reconhecer o direito do doente à sua informação de saúde significa cortar amarras com a velha máxima hipocrática de que o doente não tem capacidade para conhecer a sua informação de saúde, e que a mesma só deve estar na disponibilidade e controlo do médico (17) ${ }^{37}$.

E neste sentido, vem a Constituição da República Portuguesa, no seu artigo 35.․, sob a epígrafe "utilização da informática" 38 , dispor, no n. ${ }^{\circ} 1$, que "todos os cidadãos têm direito de acesso aos dados informatizados que lhe digam respeito, podendo exigir a sua rectificação e actualização, e o direito de conhecer a finalidade a que se destinam, nos termos da lei". No entanto, os dados relativos à vida privada - que englobam os dados de saúde - só poderão ser objeto de tratamento caso o titular desses dados o tenha

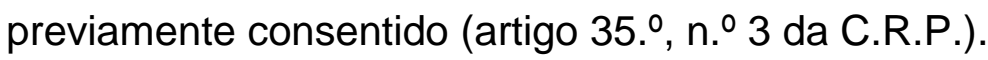

\footnotetext{
${ }^{37}$ A propósito de um estudo sobre o acesso dos pacientes aos dados de saúde um grupo de investigadores conclui que "most patients and healthcare professionals seem to be unanimous in their belief that the impact of patients' access to their medical records is positive for both. Nor only are there some real benefits in the patient accessing his/her medical record but also new technologies can help improving and supporting this access.",

38 Não obstante a epígrafe deste artigo, também os ficheiros manuais gozam de idêntica proteção (cfr. n.ำ 7 do artigo 35.ำ da Constituição da República Portuguesa).
} 


\section{O consentimento informado enquanto passo indispensável para a afirmação da liberdade terapêutica}

Assente que existe um direito à informação, quem é o titular dessa informação e que este pode aceder à sua informação de saúde dado que esta faz parte da sua vida privada, resta-nos passar ao segundo passo do consentimento: a obtenção de um consentimento livre. O consentimento informado, como vimos, é um processo classicamente dividido em duas partes: a informação e o livre consentimento.

A informação, como verificámos, deverá ser disponibilizada numa linguagem clara e acessível seja na forma verbal ou na forma escrita, baseada na legis artis e isenta quanto a juízos de valor. Deve ser suficientemente precisa para alcançar o objetivo de esclarecer sobre o diagnóstico, alcance, envergadura e consequências - diretas e indiretas, positivas e negativas - da intervenção proposta, obrigando-se a uma razoabilidade temporal que emana da necessidade da pessoa precisar de um tempo de reflexão, e deixando margem para o contraditório que poderá vir a surgir de uma segunda opinião a que todos têm direito $^{39}$. É fundamental para avaliar qualitativamente a informação assegurar que o doente compreendeu a mensagem que foi transmitida.

O consentimento refere-se, assim, à decisão voluntária de aceitação e à autorização propriamente dita (18) para o ato proposto.

Assim, é, pois, de fundamental importância que o paciente tenha conhecimento da terapêutica que Ihe está a ser ministrada. E se, como sabemos, a informação deve estar na base de todos os atos médicos, pautando-se a conduta do praticante em qualquer intervenção terapêutica pela própria necessidade de existir um consentimento informado, por maioria de razão, torna-se fundamental que esse consentimento informado se verifique neste procedimento. (5) ${ }^{40}$

O consentimento informado é um passo crucial no desenvolvimento do ato médico e está, sem dúvida, consagrado no direito português (10) nos seus vários planos, seja constitucional, civil, penal ou deontológico, sendo que a necessidade de obter tal consentimento tem o seu fundamento na proteção dos direitos à autodeterminação e à

\footnotetext{
39 Artigo 7. da Carta dos Direitos e Deveres dos Doentes (www.portaldasaude.pt/portal/conteudos/informacoes+uteis/direitos+deveres/direitosdeveresdoente.htm) 40 "The doctrine of informed consent obliges physicians to provide patients with material information about proposed treatments, alternatives, and potential risks and benefits of each."
} 
integridade física e moral do ser humano. De resto, esta preocupação tem sido manifestada em Portugal, muito embora não exista, ainda, regulamentação acerca desta prática. A verdade é que a Norma 15/2013 da Direção Geral de Saúde, relativa ao consentimento informado, livre e esclarecido, indica que este deve ser obrigatoriamente prestado por escrito quando, entre outros casos, em causa esteja o uso off-label de medicamentos de dispensa hospitalar. ${ }^{41}$ Não nos parece, de resto, exagerada esta medida, sobretudo tratando-se de situações mais complexas.

Acreditamos que ao paciente sujeito a um uso off-label de um medicamento tem de ser dado um esclarecimento detalhado da terapêutica que lhe está a ser ministrada. O doente tem o direito de saber que o medicamento que lhe está a ser prescrito não está oficialmente aprovado para o uso, indicando-se-Ihe a ausência de experimentação e aprovação pelas autoridades nacionais e, nessa medida, a falta de certeza e segurança.

É imperativo que se exponham, tendo em atenção o paciente e as suas características, quais os riscos e benefícios que daquela prescrição poderão advir. $\mathrm{O}$ médico deverá referir os fundamentos que o levam a crer tratar-se de uma opção viável, esclarecendo o estado da arte e apresentando razões para a existência de expectativa no benefício direto, recorrendo, se necessário, à apresentação de casos de sucesso. Além disso, parece-nos, este profissional deverá, ainda, apresentar as diferentes alternativas ou, em princípio, a sua ausência - e as consequências que poderão ser previsíveis ( 5) (19).

Em alguns casos, os pacientes poderão, mesmo, pedir informações adicionais, e procurar conhecer a literatura e documentação onde o médico assenta a sua convicção para prescrever a terapia em questão. Só assim poderá existir um efetivo consentimento para que o médico proceda da forma mais correta e só assim se poderá acautelar que uma prescrição off-label não caia numa prática que viole as leges artis e que frustre os princípios basilares do direito da medicina.

Deste modo se poderá compreender que, mesmo em situações mais excepcionais, onde a evidência que suporta a prescrição off-label não seja muito elevada, o médico o possa fazer para aquele doente em particular, na situação em concreto. Se o estado do paciente for periclitante e a condição rara, se existirem fortes motivos para se crer num

41 Cfr. "Norma da Direção-Geral de Saúde N ․o 015/2013, de 3/10/2013, actualizada a 14/10/2014 (www.dgs.pt) 
potencial benefício, que ultrapasse um potencial risco, se não existir qualquer outra terapia e se o doente se encontrar completamente informado e, para tal, fornecer o seu consentimento, então, acreditamos que o uso off-label não será inapropriado.

Nessa medida, a prescrição off-label está, obviamente, dependente de um juízo medico e, consequentemente, o paciente terá de acreditar que os benefícios diretos da utilização daquele medicamente serão superiores aos riscos existentes e inerentes a um uso não autorizado.(19) O médico deverá ser particularmente cuidadoso a registar no processo clínico do paciente todo este processo, nomeadamente deve registar de forma clara e compreensível o processo de esclarecimento e a decisão plenamente esclarecida do paciente.

Infelizmente, na Europa, como não existe nenhuma orientação legislativa, para os países membros, quando à prescrição off-label cada Estado-membro regula a matéria de forma diferente criando uma grande disparidade na Europa. Na verdade, existem países como Espanha e Itália, que regulam, especificamente, o uso off-label e o respectivo consentimento informado. No entanto, a grande maioria dos países europeus não dispõem de regulamentação. Certo é que o consentimento informado deve ser considerado um direito fundamental europeu. Cândido Garcia Molyneaux e Peter Bogaert (20) explicam-nos que

o consentimento informado se tornou um Direito Fundamental Europeu. A Convenção de Oviedo exige o consentimento informado do paciente para qualquer ato medico. Neste sentido, e muito embora nem todos os Estados membros terem ratificado a Convenção de Oviedo, o Tribunal Europeu dos Direitos do Homem tem garantido a protecção do direito de consentir de forma livre, clara e informada, assegurada pelo artigo $5 .^{\circ}$ da Convenção, assumindo que a sua ausência constitui uma violação do direito ao respeito pela vida privada, previsto na Convenção Europeia dos Direitos do Homem, pela qual todos os Estados-membros estão obrigados.

\section{Consentimento informado escrito na prescrição off-label e o processo clínico: dificuldades na utilização do processo clinico electrónico}

Como já referimos supra a lei portuguesa exige, expressamente, o consentimento escrito para "o uso off-label de medicamentos de dispensa hospitalar". Esta exigência é, 
como também referimos, recente e feita através de uma norma - soft law - da Direção Geral de Saúde ${ }^{42}$.

Contudo, em rigor, obter o consentimento, quer oral, quer escrito, do paciente é apenas uma das partes do processo do consentimento informado, livre e esclarecido. E, efetivamente, a parte mais importante é fornecer ao paciente a informação adequada - nos termos a que anteriormente nos referimos - sobre o uso off-label daquele concreto medicamento, para o seu concreto caso, permitindo, assim, que exista uma decisão verdadeiramente informada.

Mas, assim, neste caso concreto do uso off-label, estamos perante um problema acrescido: os desenvolvimentos e, especificamente, o processo clínico eletrónico. Como conciliar esta exigência de um consentimento informado escrito com o uso do processo clínico e demais plataformas eletrónicas?

De facto, hoje em dia, a comunidade médica usa dispositivos eletrónicos e informáticos para fornecer a informação que, geralmente, está contida no documento para obtenção do consentimento informado escrito, comprovando, aí, a decisão do paciente.

O processo eletrónico para o consentimento informado poderá usar uma interface interativa como a Plataforma da Dados da Saúde ${ }^{43}$ o que poderá facilitar a capacidade do paciente para aceder e compreender a informação fornecida.

Além disso, estes processos eletrónicos poderão permitir uma mais fácil e célere notificação dos pacientes de qualquer aperfeiçoamento ou acréscimo de informação relativo ao ato para o qual se procura o consentimento informado.

No entanto, este tipo de procedimento eletrónio aporta algumas dúvidas e obstáculos como, por exemplo, o facto de sendo o consentimento escrito exigido, o uso destes meios tecnológicos e digitais permitirão o uso de assinaturas digitais? Ou deverá ser fornecida uma cópia digital? Os pacientes deverão ser informados, dos riscos existentes ao armazenar e consultar os documentos relativos ao consentimento informado num

\footnotetext{
42 Guideline 15/2014 da Direção Geral de Saúde (General Health Directorate). Cfr. "Norma da Direção-Geral de Saúde N. $.015 / 2013$, de 3/10/2013, actualizada a 14/10/2014 (www.dgs.pt)

43 "A Plataforma de Dados da Saúde (PDS) é uma plataforma web, que disponibiliza um sistema central de registo e partilha de informação clínica de acordo com os requisitos da Comissão Nacional de Proteção de Dados. A plataforma permite o acesso a informação dos cidadãos que tenham número de utente do Serviço Nacional de Saúde (SNS), aos profissionais de saúde em diversos pontos do SNS (hospitais, urgências, cuidados primários, rede nacional de cuidados continuados), sem os deslocar do local seguro onde agora estão guardados. Este acesso pode ser auditado e gerido pelo próprio utente através do Portal do Utente." (http://spms.min-saude.pt/2013/11/pds-plataforma-de-dados-da-saude/)
} 
dispositivo eletrónico pessoal, sobretudo porque esse dispositivo eletrónico poderá ser partilhado com outras pessoas, ou poderá ainda ser perdida informação ou, pior, "pirateada"... Ao contrário das cópias em papel, que o paciente pode recusar-se a levar consigo ou pode destruir, as cópias enviadas diretamente para o dispositivo eletrónico pessoal do paciente poderão não ser removidos permanentemente... Portanto, o sistema eletrónico que suporta o consentimento informado eletrónico deve ser seguro com acesso restrito e deve incluir métodos para garantir a confidencialidade sobre a identidade do paciente, tratamentos de saúde e informações pessoais após a obtenção do consentimento informado ${ }^{44}$.

\section{Conclusão}

A prescrição off-label é uma realidade, com a qual nos deparamos diariamente, manifestamente complexa e que merece atenção e precaução.

$\mathrm{Na}$ Europa, embora a legislação europeia regule as autorizações de introdução no mercado e comercialização de medicamentos, ainda não existe uma regulação específica para o uso off-label. Apesar de uma série de iniciativas de regulamentação na União Europeia, mantém-se uma situação insatisfatória, mesmo após múltiplas críticas dos profissionais que lidam com este tipo de prescrição (21).

Não existem, contudo, quaisquer dúvidas de que é imprescindível esclarecer, detalhadamente, o paciente para que se possa obter o consentimento informado para uma prescrição off-label, sendo que este consentimento exige informação, documentação e, finalmente, uma assinatura. Claro está que isto beneficia tanto o paciente, definindo expectativas e, assim, limitar, também, a responsabilidade do médico prescritor. Automatizar o processo de consentimento poderá reduzir o risco de processos de responsabilidade médica, que surgem a partir da discrepância entre as expectativas do paciente e do resultado do tratamento (22).

O futuro certamente trará, cada vez mais, avançadas tecnologias para o sector da saúde. Um exemplo claro são as plataformas europeias para reportar os eventos adversos (como o Eudravigilance) ou, num contexto menor, mas mais concreto, a já mencionada

44 Use of Electronic Informed Consent in Clinical Investigations-Questions and Answers," Guidance for Industry, FDA, March 9, 2015, (elC Guidance) available electronically at:

http://www.fda.gov/ucm/groups/fdagov-public/@fdagov-drugsgen/documents/document/ucm436811.pdf 
Plataforma de Dados de Saúde, em Portugal. Caberá, pois, às autoridades desenvolver os vários mecanismos, fazendo os ajustes necessários e preparando e educando não só os profissionais de saúde, como também os pacientes para estas novas realidades.

\section{Referências}

1. Zimney, E. Is Your Prescription Off-Label?, Dr. Z's Medical Report - HealthTalk Disponível em: http://blog.healthtalk.com/zimney/is-your-prescription-off-label/. Acesso em 7 jul 2016

2. Dresser, R; Frader, J, Off-label Prescribing: A Call for Heightened Professional and Government Oversight, The Journal of Law, Medicine \& Ethics, 37 (3): 476-496. 2009

3. Riley, JB., Basilius, P. A, Physicians' liability for off-label prescriptions, Hematology \& Oncology News \& Issues, may/june 2007. Disponivel em: www.honionline.com. Acesso em 7 jul 2016.

4. Andrade, MC, anotação ao artigo 150.․, Comentário Conimbricense do Código Penal, 2. a Ed. Coimbra: Coimbra Editora, 2012

5. Mehlman, MJ., Off-Label Prescribing. Disponível em: http://www.thedoctorwillseeyounow.com/content/bioethics/art1971.html. Acesso em 7 jul 2016

6. Van Den Brink, $\mathrm{H}$; Cheron, $\mathrm{C}$, Les médicaments pédiatriques, un règlement européen incitatif et sécurisant, Revue Générale de Droit Médical, nº 25, Les Études Hospitalières Éditions, 2007

7. Di Paolo, E., et al., "Unlicensed and off-label drug use in a Swiss paediatric university hospital", Swiss Medical Weekly, n. 136, 2006

8. Rebecq, G, La prescription médical, Aix-en-Provence: Presses Universitaires d'AixMarseille, 1998

9. Costa, JF, Em redor da noção de acto médico Revista de Legislação e Jurisprudência,

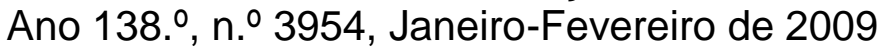

10. Oliveira, G; Pereira, AD, Consentimento Informado - Relatório Final, Entidade Reguladora da Saúde, 2009. Disponível em www.ers.pt.Acesso em 7 jul 2016

11. Rodrigues, JV, O consentimento informado para o acto médico no ordenamento jurídico português, Centro de Direito Biomédico, Coimbra, Coimbra Editora, 2001

12. Oliveira, G, Estrutura Jurídica do Acto Médico. In Temas de Direito da Medicina, Centro de Direito Biomédico, Coimbra Editora, Coimbra,.2012 
13. Canotilho, J. Gomes e Moreira, V, Constituição da República Portuguesa Anotada, 3. ${ }^{\mathrm{a}}$ edição, Coimbra, 1993.

14. Romeo-Casabona, C. Anonymization and Pseudonymisation: the legal Framework at a european level. In Beyleveld ; Townend ;S. Rouillé-Mirza ; Wright J. (edit). The data protection directive and medical reserach across europe, Ashgate, England, 2004.

15. Pinto, PM. A protecção da vida privada e a Constituição. In Boletim da Faculdade de Direito, v. LXXVI, 200․

16. Oliveira, G. O fim da 'arte silenciosa. In Temas de Direito da Medicina, Coimbra Editora, 2005.

17. Ferreira, Ana; Correia, et alli, Why facilitate patient Access to medical records, Disponível em www.cintesis.med.up.Acesso em 7 jul 2016

18. Beauchamp TL.; Childress, JF. Principles of Biomedical Ethics, 5th ed Oxford University Press. 2001

19. Melonas, J., Preventing and Reducing Professional Liability Risk Related to Psychopharmacology. Disponivel em http://www.psychiatrictimes.com/showArticle.jhtml?articleld=175803689. Acesso em 7 jul 2016

20. Molyneaux, CG ; Bogaert, $P$, The need for informed consent in off-label use in the EU legal analysis. Disponível em: www.regulatoryaffairspharma.com. Acesso em 7 jul 2016

21. Lenk, C; Duttge, G Ethical and legal framework and regulation for off-label use: European perspective. Therapeutics and Clinical Risk Management, v 10, 2014

22. Stemp, MW., Informed consent: How technology can improve the process, Dermatology World, oct, 2014.

\section{Como citar este artigo:}

Barbosa C, Matos MF. Prescrição off-label, direito à informação, consentimento informado e processo clinico eletrônico no direito português. Revista Cadernos Ibero-Americanos de Direito Sanitário. 2016 jul./set, 5(3):157-179 